\title{
Solitary Wave Solution of the Two-Dimensional Regularized Long-Wave and Davey-Stewartson Equations in Fluids and Plasmas
}

\author{
Omar H. El-Kalaawy, Rafat S. Ibrahim \\ Department of Mathematics, Faculty of Science, Beni-Suef University, Beni-Suef, Egypt \\ Email: ohkalaawy7@gmail.com
}

Received June 9, 2012; revised July 6, 2012; accepted July 13, 2012

\begin{abstract}
This paper investigates the solitary wave solutions of the (2+1)-dimensional regularized long-wave (2DRLW) equation which is arising in the investigation of the Rossby waves in rotating flows and the drift waves in plasmas and $(2+1)$ dimensional Davey-Stewartson (DS) equation which is governing the dynamics of weakly nonlinear modulation of a lattice wave packet in a multidimensional lattice. By using extended mapping method technique, we have shown that the 2DRLW-2DDS equations can be reduced to the elliptic-like equation. Then, the extended mapping method is used to obtain a series of solutions including the single and the combined non degenerative Jacobi elliptic function solutions and their degenerative solutions to the above mentioned class of nonlinear partial differential equations (NLPDEs).
\end{abstract}

Keywords: Exact Solitary Solutions; Extended Mapping Method; Two Dimension Regularized Long Wave and Davey-Stewartson Equations; Jacobi Elliptic Functions

\section{Introduction}

In the recent years, seeking exact solutions of nonlinear partial differential equations (NLPDEs) is of great significance, since the nonlinear complex physical phenomena related to the NLPDEs are involved in many fields from physics (plasma physics, optical fibers, solid state physics, nonlinear optics and so on), fluid mechanics, biology, chemistry kinetics, geochemistry and engineering. As mathematical models of the phenomena, the investigation of exact solutions of NLPDEs will help one to understand the mechanism that governs these physical models or to better provide knowledge of the physical problem and possible applications. To this aim, a vast variety of powerful and direct methods for finding the exact significant solutions of the NLPDEs through it is rather difficult have been derived. Some of the most important methods are Hirota's dependent variable transformation [1], the Bäcklund transformations (BTs) [2], the inverse scattering transformation [3], Painlevé expansions [4], Jacobi elliptic function expansion method [5-7], the homogenous balance method [8], the linearized transformation method [9-11], the F-expansion method $[12,13]$, Fan-sub-equation method, extended and modified extended Fan-sub equation method [14-18], the tanh-function method and extended tanh-function method [19-21], the tanh-sech method [22], the sine-cosine me- thod [23,24], variational iteration method [25], homotopy perturbation method [26], the $\left(G^{\prime} / G\right)$-expansion method [27-29] and several ansatz methods [30-34].

The Frobenius integrable decompositions (FIDs) and rational function transformations (RFTs) are used to construct exact solutions to NLPDEs with BTs and auto BTs [35-40]. Recently, Ma et al. [37] presented Frobenius integrable decompositions (FIDs) for two classes of nonlinear evolution equations (NEEs) with logarithmic derivative BTs in soliton theory. The discussed NEEs are transformed into systems of Frobenius integrable ODEs with cubic nonlinearity. You et al. [41] obtained two classes of PDEs with variable coefficients possessing FIDs, including the $\mathrm{KdV}$ and the potential $\mathrm{KdV}$ equation, the Boussinesq equation, and the generalized BBM equation. The RFTs method is very suitable for an easier and more effective handling of the solution process of nonlinear equations, unifying the existing solution methods mentioned above. Its key point is to find rational solutions to variable-coefficient ODEs transformed from given NLPDEs, together with an auto-BT.

The main aim of this paper is to find exact solitary solutions of $(2+1)$ dimensional regularized long wave (2DRLW) and (2+1) Davey-Stewartson (DS) equations. The paper is organized as follows: This introduction is presented in Section 1. In Section 2 we give a description of the extended mapping method and we apply this me- 
thod to the $(2+1)$ regularized long wave equation and the Davey-Stewartson equation. In Section 3, some conclusions are given.

\section{The Extended Mapping Method}

We are given a NLPDE for $\phi(\xi, \tau)$ in the form

$$
F\left(\phi, \phi_{\tau}, \phi_{\xi}, \phi_{\xi \xi}, \phi_{\tau \tau}, \phi_{\tau \xi}, \cdots\right)=0 \text {. }
$$

Introducing the similarity variable $\zeta=k(\xi-\omega \tau)$, then the function $\phi$ satisfies the following ordinary differential equation (ODE)

$$
H\left(\phi, \phi_{\zeta}, \phi_{\zeta}, \phi_{\zeta \zeta}, \phi_{\zeta \zeta \zeta}, \cdots\right)=0 \text {. }
$$

By virtue of the extended mapping method we assume that the solution of Equation (2) in the form

$$
\begin{aligned}
\phi(\zeta) & =a_{0}+\sum_{i=1}^{N}\left(a_{i} f^{i}(\zeta)+b_{i} f^{-i}(\zeta)\right) \\
& +\sum_{i=2}^{N} c_{i} f^{-i}(\zeta) f^{\prime}(\zeta)+\sum_{i=-1}^{-N} d_{i} f^{i}(\zeta) f^{\prime}(\zeta),
\end{aligned}
$$

where $N$ in Equation (3) is a positive integer that can be determined by balancing the nonlinear term(s) with the highest derivative term in Equation (2) and $a, a_{i}, b_{i}$, $c_{i}$ and $d_{i}$ are constants to be determined. The function $f(\zeta)$ satisfies the nonlinear ODE

$$
\left(\frac{\mathrm{d} f}{\mathrm{~d} \zeta}\right)^{2}=q_{0}+q_{2} f^{2}(\zeta)+q_{4} f^{4}(\zeta),
$$

where $q_{0}, q_{2}$ and $q_{4}$ are constants. Substituting Equation (3) with Equation (4) into the ODE Equation (2) and setting the coefficients of the different powers of $f^{i}(\zeta)$ to zero yields a set of algebraic equations for $a_{0}$, $a_{i}, b_{i}, c_{i}, d_{i}$ and $k$. Solving the algebraic equations by use of Maple or Mathematica, we have $a_{0}, a_{i}, b_{i}$, $c_{i}, d_{i}$ and $k$ expressed by $q_{0}, q_{2}, q_{4}$. Substituting the obtained coefficients into Equation (3), then concentration formulas of travelling wave solutions of the NLPDE Equation (1) can be obtained. Selecting the values of $q_{0}, q_{2}, q_{4}$ and the corresponding JEFs $f(\zeta)$ from the table in Appendix and substituting them into the concentration formulas of solutions to obtain the explicit and exact JEF solutions of Equation (1). Various solutions of Equation (4) were constructed using JEFs, and these results were exploited in the design of a procedure for generating solutions of NLPDEs. The JEFs $s n \zeta=s n(\zeta, m)$, $c n \zeta=c n(\zeta, m)$ and $d n \zeta=d n(\zeta, m)$ where $m(0<m<1)$ is the modulus of the elliptic function, are double periodic and posses the following properties

$$
\begin{aligned}
& s n^{2}(\zeta)+c n^{2}(\zeta)=1, \quad d n^{2}(\zeta)+m^{2} s n^{2}(\zeta)=1, \\
& d n^{2} \zeta=m^{2} c n^{2} \zeta+\left(1-m^{2}\right), \quad(\operatorname{sn} \zeta)^{\prime}=c n(\zeta) d n(\zeta), \\
& (c n \zeta)^{\prime}=-\operatorname{sn}(\zeta) d n(\zeta),
\end{aligned}
$$

$$
(d n \zeta)^{\prime}=-m^{2} \operatorname{sn}(\zeta) c d n(\zeta)
$$

In addition when $m \rightarrow 1$, the functions $s n \zeta, c n \zeta$ and $d n \zeta$ degenerate as $\tanh \zeta, \sec h \zeta$ and $\sec h \zeta$, respectively, while when $m \rightarrow 0, s n \zeta, c n \zeta$ and $d n \zeta$ degenerate as $\sin \zeta, \cos \zeta$ and 1 , respectively. So, we can obtain hyperbolic function solutions and trigonometric function solutions in the limit cases when $m \rightarrow 1$ and $m \rightarrow 0$. Some more properties of JEFs can be found in [33].

\section{1. (2+1) Regularized Long Wave Equation}

Let us first consider the regularized long wave equation:

$$
\begin{aligned}
& u_{t}+\alpha_{1} u_{x}+\alpha_{2} u_{y}+\beta_{1} u u_{x}+\beta_{2} u u_{y} \\
& -\gamma_{1} u_{x x t}-\gamma_{2} u_{y y t}=0
\end{aligned}
$$

have been reported in $[42,43]$ where the coefficients $\beta_{1}$, $\beta_{2} \neq 0, \quad \gamma_{1}>0, \quad \gamma_{2}, \alpha_{1}$ and $\alpha_{2}$ are all constants. Equation (5) is related to the drift waves in plasma and the Rossby waves in rotating fluids [44]. To look for travelling wave solution of Equation (2.5), we make transformation $u(x, y, t)=u(\xi), \quad \xi=k(l x+n y-\omega t) \quad$ and change Equation (5) into the form

$$
\alpha u^{\prime}+\beta u u^{\prime}+\gamma k^{2} u^{\prime \prime \prime}=0,
$$

where $\alpha=\left(-\omega+l \alpha_{1}+\alpha_{2} n\right), \quad \beta=\left(l \beta_{1}+\beta_{2} n\right)$, and $\gamma=\omega\left(l^{2} \gamma_{1}+\gamma_{2} n^{2}\right)$. Integrating once with respect to $\xi$ and setting the integration constant equal to zero, one has

$$
\alpha u+\frac{\beta}{2} u^{2}+\gamma k^{2} u^{\prime \prime}=0 .
$$

Balancing $u^{2}$ with $u^{\prime \prime}$ gives the leading order $N=2$. So take the anastz

$$
\begin{aligned}
u(\zeta) & =a_{0}+a_{1} f(\zeta)+a_{2} f^{2}(\zeta)+\frac{b_{1}}{f(\zeta)}+\frac{b_{2}}{f^{2}(\zeta)} \\
& +c_{2} f^{\prime}(\zeta)+\frac{d_{1} f^{\prime}(\zeta)}{f(\zeta)}+\frac{d_{2} f^{\prime}(\zeta)}{f^{2}(\zeta)}
\end{aligned}
$$

where $a_{0}, a_{1}, a_{2}, b_{1}, b_{2}, c_{2}, d_{1}, d_{2}$ and $k$ are constants and need to be determined, $f(\zeta)$ is a solution of Equation (4). Substituting Equation (4) and Equation (8) into Equation (7) and setting the coefficients of $f^{i}(\zeta)$, to zero, we get a system of nonlinear equations for $a_{0}, a_{1}, a_{2}, b_{1}, b_{2}, c_{2}, d_{1}, d_{2}$ and $k$. Solving this system by use of Mathematica, we obtain:

Case

$$
\begin{aligned}
& a_{1}=a_{2}=b_{1}=c_{2}=d_{1}=d_{2}=0, \\
& a_{0}=-\frac{\alpha}{\beta}\left(1+\frac{q_{2}}{\sqrt{q_{2}^{2}+3 q_{0} q_{4}}}\right)
\end{aligned}
$$




$$
b_{2}=-\frac{3 \alpha q_{2}}{\beta \sqrt{q_{2}^{2}+3 q_{0} q_{4}}}, k= \pm \frac{1}{2} \sqrt{\frac{\alpha}{\beta}} \frac{1}{\left(q_{2}^{2}+3 q_{0} q_{4}\right)^{1 / 4}} .
$$

\section{Case 1}

$$
\begin{aligned}
a_{1} & =b_{1}=c_{2}=d_{1}=d_{2}=0, a_{0}=-\frac{\alpha}{\beta}\left(1+\frac{q_{2}}{\sqrt{q_{2}^{2}+12 q_{0} q_{4}}}\right) \\
a_{2} & =-\frac{3 \alpha q_{4}}{\beta \sqrt{q_{2}^{2}+12 q_{0} q_{4}}}, \quad b_{2}=-\frac{3 \alpha q_{0}}{\beta \sqrt{q_{2}^{2}+12 q_{0} q_{4}}}, \\
k & = \pm \frac{1}{2} \sqrt{\frac{\alpha}{\beta}} \frac{1}{\left(q_{2}^{2}+3 q_{0} q_{4}\right)^{1 / 4}} .
\end{aligned}
$$

\section{Case 2}

$$
\begin{aligned}
& a_{1}=b_{1}=b_{2}=d_{1}=d_{2}=0, \quad a_{0}=-\frac{\alpha}{\beta}\left(1+\frac{q_{2}}{\sqrt{q_{2}^{2}+12 q_{0} q_{4}}}\right), \\
& a_{2}=-\frac{6 \alpha q_{4}}{\beta \sqrt{q_{2}^{2}+12 q_{0} q_{4}}}, \quad c_{2}=-\frac{6 \alpha \sqrt{q_{4}}}{\beta \sqrt{q_{2}^{2}+12 q_{0} q_{4}}}, \\
& k= \pm \sqrt{\frac{\alpha}{\beta}} \frac{1}{\left(q_{2}^{2}+3 q_{0} q_{4}\right)^{1 / 4}} .
\end{aligned}
$$

\section{Case 3}

$$
\begin{aligned}
& a_{1}=b_{1}=d_{1}=0, \\
& a_{0}=-\frac{\alpha}{\beta}\left(1+q_{2}-\frac{6 \sqrt{q_{4} q_{0}}}{\sqrt{q_{2}^{2}+60 q_{2} \sqrt{q_{4} q_{0}}+132 q_{4} q_{0}}}\right) \\
& a_{2}=-\frac{6 \alpha q_{4}}{\beta \sqrt{q_{2}^{2}+60 q_{2} \sqrt{q_{4} q_{0}}+132 q_{4} q_{0}}}, \\
& b_{2}=-\frac{6 \alpha q_{0}}{\beta \sqrt{q_{2}^{2}+60 q_{2} \sqrt{q_{4} q_{0}}+132 q_{4} q_{0}}}, \\
& d_{2}=-\frac{6 \alpha \sqrt{q_{0}}}{\sqrt{q_{2}^{2}+60 q_{2} \sqrt{q_{4} q_{0}}+132 q_{4} q_{0}}}, \\
& c_{2}=-\frac{6 \alpha \sqrt{q_{4}}}{\beta \sqrt{q_{2}^{2}+60 q_{2} \sqrt{q_{4} q_{0}}+132 q_{4} q_{0}}}, \\
& k= \pm \sqrt{\left.\frac{\alpha}{\beta} \frac{1}{\left(q_{2}^{2}+60 q_{2} \sqrt{q_{4} q_{0}}+132 q_{4} q_{0}\right.}\right)^{1 / 4}} .
\end{aligned}
$$

If $q_{0}=1, \quad q_{2}=-\left(1+m^{2}\right), \quad q_{4}=m^{2}, \quad F(\zeta)=n s^{2} \zeta$, this yields the exact solutions of Equation (7) as follows:

$$
u(x, y, t)=-\frac{\alpha}{\beta \sqrt{\left(-\left(1+m^{2}\right)\right)^{2}+3 m^{2}}}\left(\sqrt{\left(-\left(1+m^{2}\right)\right)^{2}+3 m^{2}}-\left(1+m^{2}\right)+3 n s^{2} \pm\left[\sqrt{\frac{\alpha}{4 \gamma \sqrt{\left(-\left(1+m^{2}\right)\right)^{2}+3 m^{2}}}} \xi\right] .\right.
$$

when $m \rightarrow 1$, the solitary wave solutions of Equation (5) are obtained as follows:

$$
u(x, y, t)=-\frac{\left(-\omega+l \alpha_{1}+n \alpha_{2}\right)}{\sqrt{7}\left(\beta_{1} l+\beta_{2} n\right)}\left(\sqrt{7}-2+3 \operatorname{coth}^{2}\left[\sqrt{\frac{\left(-\omega+l \alpha_{1}+\alpha_{2} n\right)}{4 \omega \sqrt{7}\left(\gamma_{1} l^{2}+\gamma_{2} n^{2}\right)}}(l x+n y-\omega t)\right]\right)
$$

We have represented this solution for a set of parameter values in Figure 1(a).
When $m \rightarrow 0$, the triangular periodic solutions of Equation (5) are obtained as follows:

$$
u(x, y, t)=-\frac{\left(-\omega+l \alpha_{1}+n \alpha_{2}\right)}{\left(\beta_{1} l+\beta_{2} n\right)}\left(3 \csc ^{2}\left[\sqrt{\frac{\left(-\omega+l \alpha_{1}+\alpha_{2} n\right)}{4 \omega\left(\gamma_{1} l^{2}+\gamma_{2} n^{2}\right)}}(l x+n y-\omega t)\right]\right) .
$$

We have represented this solution for a set of parameter values in Figure 1(b).

$$
\begin{aligned}
& \text { If } q_{0}=1, \quad q_{2}=-\left(1+m^{2}\right), \quad q_{4}=m^{2}, F(\zeta)=\operatorname{sn} \zeta \\
& \qquad(x, y, t)=-\frac{\left(-\omega+l \alpha_{1}+n \alpha_{2}\right)}{\left(\beta_{1} l+\beta_{2} n\right)}\left(2+3\left(\tanh \left[\frac{1}{4} \sqrt{\frac{\alpha}{\gamma}}(l x+n y-\omega t)\right]\right)^{2}+3 \operatorname{coth}\left[\frac{1}{4} \sqrt{\frac{\alpha}{\gamma}}(l x+n y-\omega t)\right]^{2}\right) .
\end{aligned}
$$

We have represented this solution for a set of parameter values in Figure 1(c). 


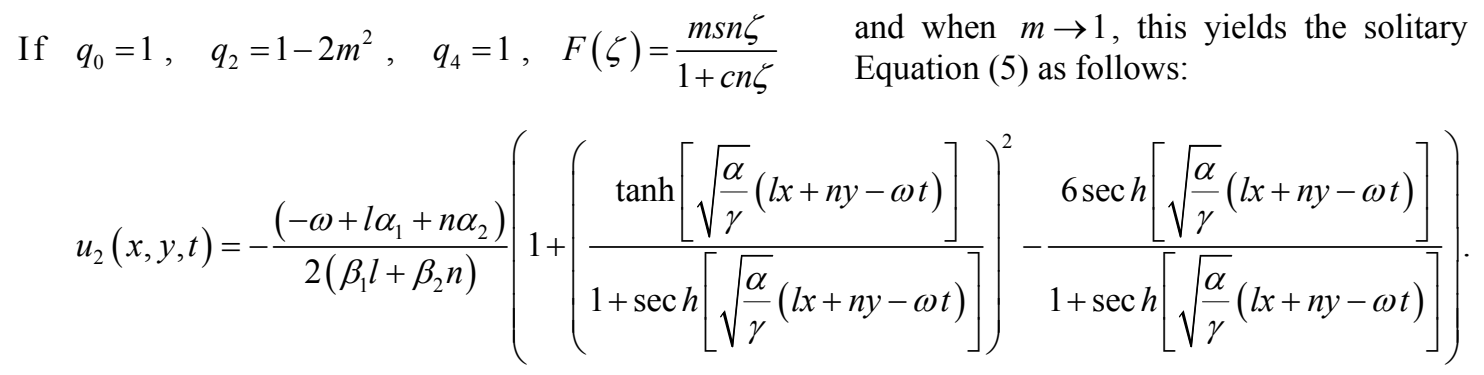

We have represented this solution for a set of parameter values in Figure 1(d).

$$
\begin{aligned}
& \text { If } q_{0}=1, \quad q_{2}=-\left(1+m^{2}\right), \quad q_{4}=m^{2}, \quad F(\zeta)=s n \zeta \\
& \quad u_{3}(x, y, t)=-\frac{\left(-\omega+l \alpha_{1}+n \alpha_{2}\right)}{4\left(\beta_{1} l+\beta_{2} n\right)}\left(2+12 \operatorname{coth}^{2}\left[\frac{1}{2} \sqrt{\frac{\alpha}{\gamma}}(l x+n y-\omega t)\right]\right) .
\end{aligned}
$$

We have represented this solution for a set of parameter values in Figure 1(e).

\subsection{The Davey Stewartson Equation}

The dimensionless form of the DSE in $(2+1)$ dimensions, with power law nonlinearity [45]. The DS model is exactly integrable in shallow water and almost integrable in deep water. Furthermore, the model has easily identifiable coherent structures and waves, including solitons, unstable rogue-wave type modes, Stokes waves and the velocity field contains vortices,

$$
\begin{aligned}
& i u_{t}+\frac{1}{2} \alpha^{2}\left(u_{x x}+\alpha^{2} u_{y y}\right)+\beta|u|^{2} u-u v=0, \\
& v_{x x}-\alpha^{2} u_{y y}-2 \beta\left(|u|^{2} u\right)_{x x}=0 .
\end{aligned}
$$

Here, in Equations (19a) and (19b), $u$ and $v$ are the and when $m \rightarrow 1$, this yields the solitary solutions of Equation (5) as follows:

$$
\left(\Omega-\frac{1}{2} \alpha^{4}\left(1+l^{2}\right)\right) \phi+\frac{k^{2} \alpha^{2}}{2}\left(p^{2}+\alpha^{2} n^{2}\right) \phi^{\prime \prime}+\beta \phi^{3}-\phi v+i k\left(-\omega+\alpha^{3}\left(p^{2}+\alpha \ln \right)\right) \phi^{\prime}=0,
$$

$$
v^{\prime \prime}(\xi)=\frac{2 \beta p^{2}}{p^{2}-n^{2} \alpha^{2}}\left(\phi^{2}\right)^{\prime \prime}
$$

If we set

$$
\omega=\alpha^{3}\left(p^{2}+\alpha \ln \right)
$$

then Equation (21a) reduce to

$$
\begin{aligned}
& \left(\Omega-\frac{1}{2} \alpha^{4}\left(1+l^{2}\right)\right) \phi+\frac{k^{2} \alpha^{2}}{2}\left(p^{2}+\alpha^{2} n^{2}\right) \phi^{\prime \prime} . \\
& +\beta \phi^{3}-\phi v=0
\end{aligned}
$$

Integrating Equation (21b) twice, and we take the constant of integration equal zero, we have

$$
v(\xi)=\frac{2 \beta p^{2}}{p^{2}-n^{2} \alpha^{2}} \phi^{2} .
$$

Substituting Equation (24) into Equation (23) yields

$$
\begin{aligned}
& \left(\Omega-\frac{1}{2} \alpha^{4}\left(1+l^{2}\right)\right) \phi+\frac{k^{2} \alpha^{2}}{2}\left(p^{2}+\alpha^{2} n^{2}\right) \phi^{\prime \prime} \\
& +\beta \phi^{3}-\frac{2 \beta p^{2}}{p^{2}-n^{2} \alpha^{2}} \phi^{3}=0, \\
& k^{2} A \phi^{\prime \prime}+B \phi+C \phi^{3}=0,
\end{aligned}
$$

where both $\phi(\xi)$ and $v(\xi)$ are real functions, $\alpha, l$, $p, \Omega$ and $\omega$ are constants and $k$ is a constant into Equations (19a) and (19b), we have the following ODE for $\phi(\xi)$ and $v(\xi)$

where

$$
A=\frac{\alpha^{2}}{2}\left(p^{2}+\alpha^{2} n^{2}\right), \quad B=\left(\Omega-\frac{1}{2} \alpha^{4}\left(1+l^{2}\right)\right)
$$

and

$$
C=\beta-\frac{2 \beta p^{2}}{p^{2}-n^{2} \alpha^{2}} .
$$




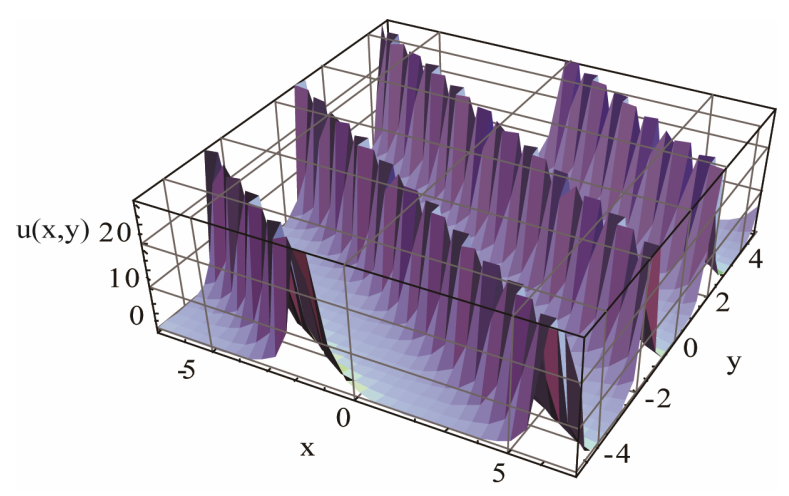

(a)

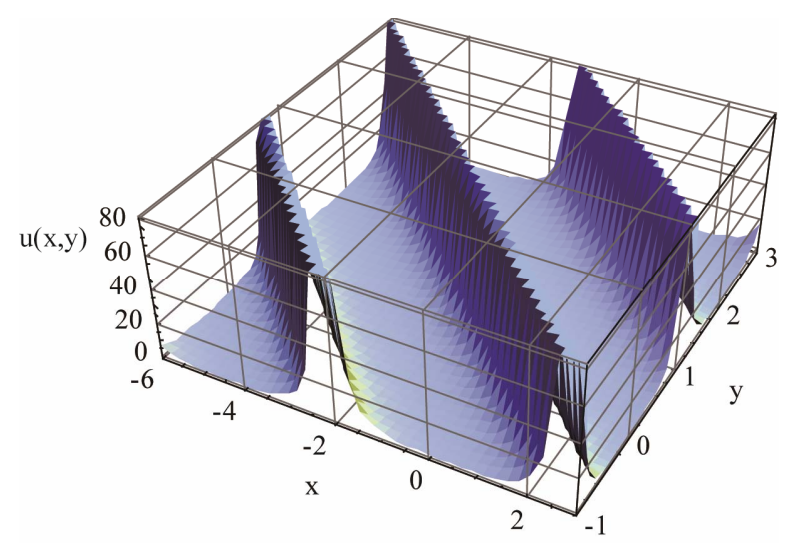

(c)

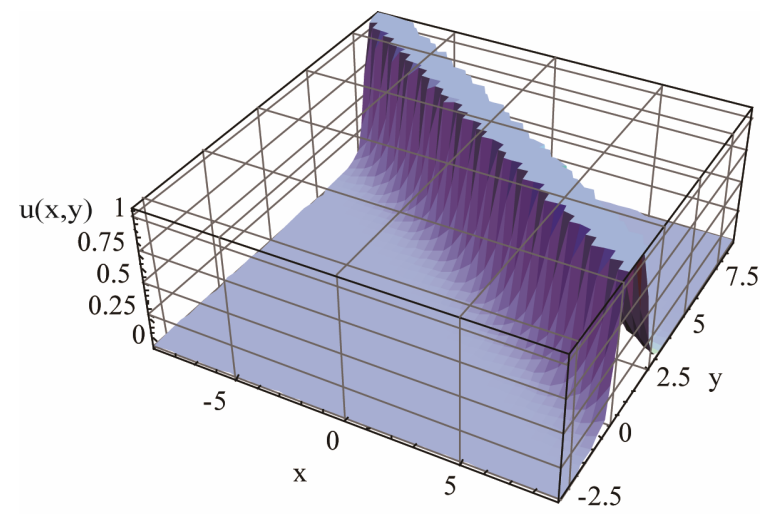

(b)

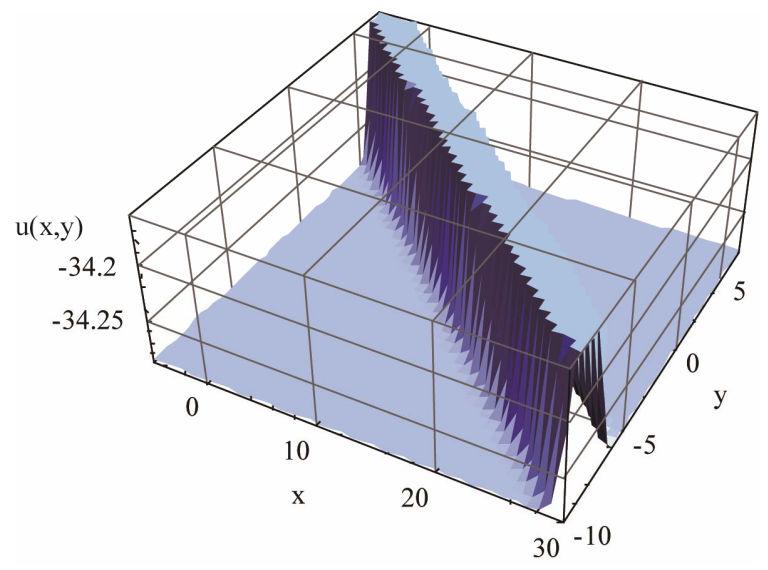

(d)

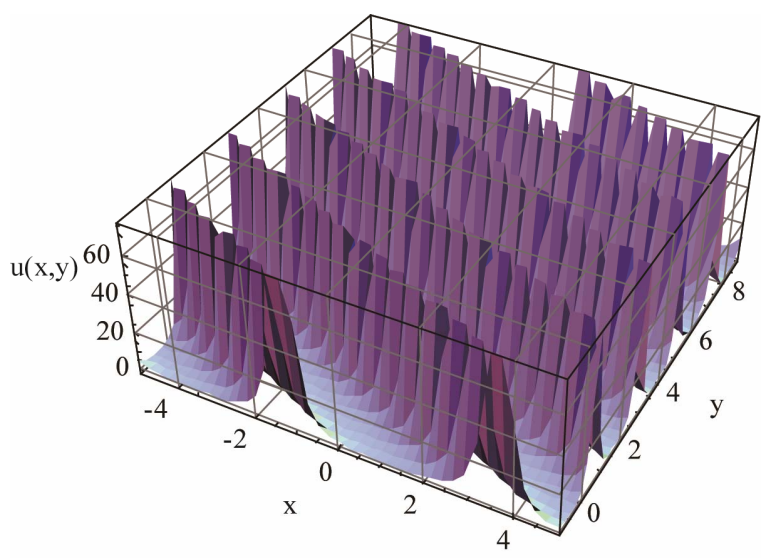

(e)

Figure 1. The solitary wave solution represented by Equations (14)-(18) for various values $\omega=0.5, a_{1}=-1, a_{2}=-2, l=0.9, m$ $=2, b_{1}=-3, b_{2}=-2, t=20, c_{1}=1, c_{2}=1.1$ for Figures 1(a)-(e) respectively.

Balancing $\phi^{3}$ with $\phi^{\prime \prime}$ gives the leading order $N=1$. So take the anastz

$$
\phi(\xi)=a_{0}+a_{1} f(\xi)+\frac{b_{1}}{f(\xi)}+\frac{d_{1} f^{\prime}(\xi)}{f(\xi)},
$$

where $a_{0}, a_{1}, b_{1}, d_{1}$ and $k$ are constants and need to be determined, $f(\zeta)$ is a solution of Equation (4) Substituting Equations (4) and (27) into Equation (26) and setting the coefficients of $f^{i}(\zeta)$, to zero, we get a system of nonlinear equations for $a_{0}, a_{1}, b_{1}, d_{1}$ and $k$. Solving this system by use of Maple, we obtain:

\section{Case}




$$
a_{0}=b_{1}=d_{1}=0, a_{1}=\sqrt{\frac{2 B q_{4}}{q_{2} C}}, k= \pm \sqrt{\frac{-B}{q_{2} A}} .
$$

\section{Case 1}

$$
a_{0}=a_{1}=d_{1}=0, b_{1}=\sqrt{\frac{2 B q_{0}}{q_{2} C}}, k= \pm \sqrt{\frac{-B}{q_{2} A}} .
$$

\section{Case 2}

$$
a_{0}=a_{1}=b_{1}=0, d_{1}=\sqrt{\frac{-B}{q_{2} C}}, k= \pm \frac{1}{2} \sqrt{\frac{2 B}{q_{2} A}} .
$$

\section{Case 3}

$$
\begin{gathered}
a_{0}=d_{1}=0, a_{1}= \pm \sqrt{\frac{2 B q_{4}}{C\left(q_{2}-6 \sqrt{q_{0} q_{4}}\right)}}, \quad \begin{array}{r}
\text { If } q_{0}=m^{2}-1, q_{2}=2-m^{2}, q_{4} \\
\text { we can obtain one Jacobian elliptic } \\
\text { Equation (26) as follows: }
\end{array} \\
u(x, y, t)=\sqrt{\frac{-2 \Omega+\alpha^{4}\left(1+l^{2}\right)}{\left(2-m^{2}\right)\left(\beta-\frac{2 \beta p^{2}}{p^{2}-n^{2} \alpha^{2}}\right)}} e^{i[\alpha x+l y-\Omega t]}\left(d n\left[\sqrt{\frac{-2 \Omega+\alpha^{4}\left(1+l^{2}\right)}{\left(2-m^{2}\right) \frac{1}{2} \alpha^{2}\left(p^{2}+\alpha^{2} n^{2}\right)} \xi}\right]\right) .
\end{gathered}
$$

when $m \rightarrow 1$, the solitary solutions of Equations (25) and (24) are obtained as follows:

$$
u(x, y, t)=\sqrt{\frac{-2 \Omega+\alpha^{4}\left(1+l^{2}\right)}{\beta-\frac{2 \beta p^{2}}{p^{2}-n^{2} \alpha^{2}}}} e^{i[\alpha x+l y-\Omega t]}\left(\sec h\left[\sqrt{\frac{-2 \Omega+\alpha^{4}\left(1+l^{2}\right)}{\left(2-m^{2}\right) \frac{1}{2} \alpha^{2}\left(p^{2}+\alpha^{2} n^{2}\right)}}(p x+n y-\omega t)\right]\right) .
$$

We have represented this solution for a set of parameter values in Figure 2(a).

$$
v(x, y, t)=-\frac{2 \beta p^{2}}{p^{2}-n^{2} \alpha^{2}} \frac{2 \Omega-\alpha^{4}\left(1+l^{2}\right)}{\left(\beta-\frac{2 \beta p^{2}}{p^{2}-n^{2} \alpha^{2}}\right)}\left(\sec h^{2}\left[\sqrt{\frac{-2 \Omega+\alpha^{4}\left(1+l^{2}\right)}{\left(2-m^{2}\right) \frac{1}{2} \alpha^{2}\left(p^{2}+\alpha^{2} n^{2}\right)}}(p x+n y-\omega t)\right]\right) .
$$

If $q_{0}=\frac{1}{4}, \quad q_{2}=\frac{m^{2}-2}{2}, q_{4}=\frac{m^{2}}{4}, F(\zeta)=\frac{s n \zeta}{1+d n \xi}, \quad \begin{aligned} & \text { this yields the solitary wave solution } \\ & \text { and (24) are obtained as follows: }\end{aligned}$

$$
u_{1}(x, y, t)=\sqrt{\frac{-B}{C}} e^{i[\alpha x+l y-\Omega t]}\left(\frac{\tanh \left[\sqrt{\frac{2 B}{A}}(p x+n y-\omega t)\right]}{1+\sec h\left[\sqrt{\frac{2 B}{A}}(p x+n y-\omega t)\right]}\right)
$$

We have represented this solution for a set of parameter values in Figure 2(b).

$$
v_{1}(x, y, t)=-\frac{2 \beta p^{2}}{p^{2}-n^{2} \alpha^{2}} \frac{B}{C}\left(\frac{\tanh \left[\sqrt{\frac{2 B}{A}}(p x+n y-\omega t)\right]}{1+\sec h\left[\sqrt{\frac{2 B}{A}}(p x+n y-\omega t)\right]}\right)^{2} .
$$


If $q_{0}=\frac{m^{2}}{4}, q_{2}=\frac{m^{2}-2}{2}, q_{4}=\frac{m^{2}}{4}$,

$F(\zeta)=\operatorname{sn} \zeta+i c n \zeta$, this yields the solitary wave solutions of Equations (25) and (24) are obtained as follows:

$$
u_{2}(x, y, t)=-i \sqrt{\frac{2 B}{C}} e^{i[\alpha x+l y-\Omega t]} \sec h\left[\sqrt{\frac{-B}{A}}(p x+n y-\omega t)\right] .
$$

We have represented this solution for a set of parameter values in Figure 2(c).

$$
v_{2}(x, y, t)=\frac{2 \beta p^{2}}{p^{2}-n^{2} \alpha^{2}} \frac{2 B}{C}\left(\sec h \sqrt{\frac{-B}{A}}(p x+n y-\omega t)\right)^{2} .
$$

If $q_{0}=m^{2}, q_{2}=-\left(m^{2}+1\right), q_{4}=1, F(\zeta)=n s \zeta$, this yields the solitary wave solutions of Equations (25) and (24) are obtained as follows:

$$
u_{3}(x, y, t)=\sqrt{\frac{-B}{4 C}} e^{i[\alpha x+l y-\Omega t]}\left(\tanh \left[\sqrt{\frac{B}{8 A}}(p x+n y-\omega t)\right]+\operatorname{coth}\left[\sqrt{\frac{B}{8 A}}(p x+n y-\omega t)\right]\right) .
$$

We have represented this solution for a set of parameter values in Figure 2(d).

$$
v_{3}(x, y, t)=-\frac{2 \beta p^{2}}{p^{2}-n^{2} \alpha^{2}} \frac{B}{4 C}\left(\tanh \left[\sqrt{\frac{B}{8 A}}(p x+n y-\omega t)\right]+\operatorname{coth}\left[\sqrt{\frac{B}{8 A}}(p x+n y-\omega t)\right]\right)^{2} .
$$

If $q_{0}=1, q_{2}=-\left(m^{2}+1\right), q_{4}=m^{2}, F(\zeta)=s n \zeta$, this yields the solitary wave solutions of Equations (25) and (24) are obtained as follows:

$$
\begin{aligned}
& u_{4}(x, y, t) \\
& \left.\left.=\sqrt{\frac{-B}{4 C}} e^{i[\alpha x+l y-\Omega t]}\right] \tanh \left[\sqrt{\frac{B}{2 A}}(p x+n y-\omega t)\right]+\operatorname{coth}\left[\sqrt{\frac{B}{2 A}}(p x+n y-\omega t)\right]+\frac{\sec h^{2}\left[\sqrt{\frac{B}{2 A}}(p x+n y-\omega t)\right]}{\tanh ^{2}\left[\sqrt{\frac{B}{2 A}}(p x+n y-\omega t)\right]}\right] .
\end{aligned}
$$

We have represented this solution for a set of parameter values in Figure 2(e).

$$
\begin{aligned}
& v_{3}(x, y, t) \\
& =-\frac{2 \beta p^{2}}{p^{2}-n^{2} \alpha^{2}} \frac{B}{4 C}\left(\tanh \left[\sqrt{\frac{B}{2 A}}(p x+n y-\omega t)\right]+\operatorname{coth}\left[\sqrt{\frac{B}{2 A}}(p x+n y-\omega t)\right]+\frac{\sec h^{2}\left[\sqrt{\frac{B}{2 A}}(p x+n y-\omega t)\right]}{\tanh ^{2}\left[\sqrt{\frac{B}{2 A}}(p x+n y-\omega t)\right]}\right)^{2} .
\end{aligned}
$$

\section{Conclusion}

In the current article, the solitary wave solutions of the two dimensional regularized long-wave equation in plasma and rotating flows simulated by using extended mapping method, and we hope these solitary waves are helpful to understand the nonlinear phenomena described by the resonant Davey-Stewartson equation in the fields like capillarity fluids. We have presented the extended mapping method to construct more general exact solu- tions of NLPDEs with the help Maple and Mathematica. This method provides a powerful mathematical tool to obtain more general exact solutions of a great many NLPDEs in mathematical physics. Applying this method to the 2DRLW and DS equations and we have successfully obtained many new exact travelling wave solutions. Through our solutions for some partial differential equations non-linear, we found lack of interest in these two methods by the specialists with the knowledge that they give an solutions more realistic than many ways, espe- 


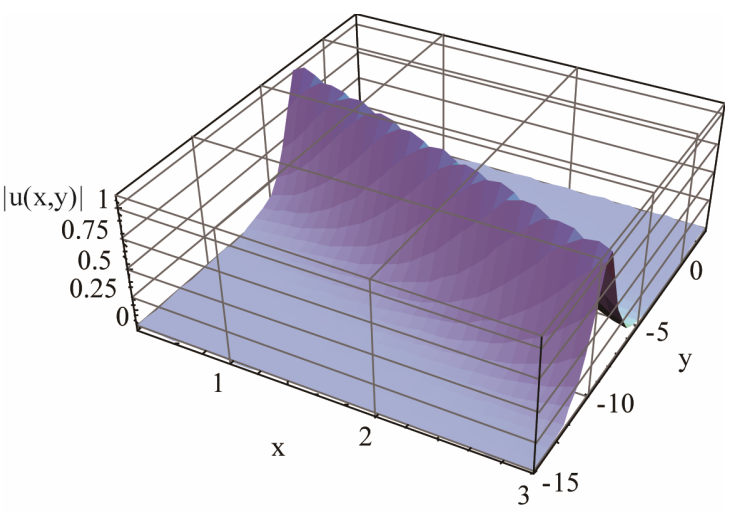

(a)

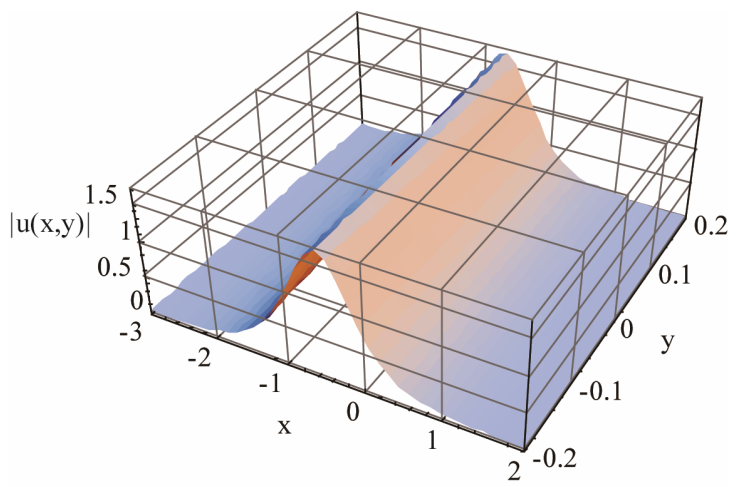

(c)

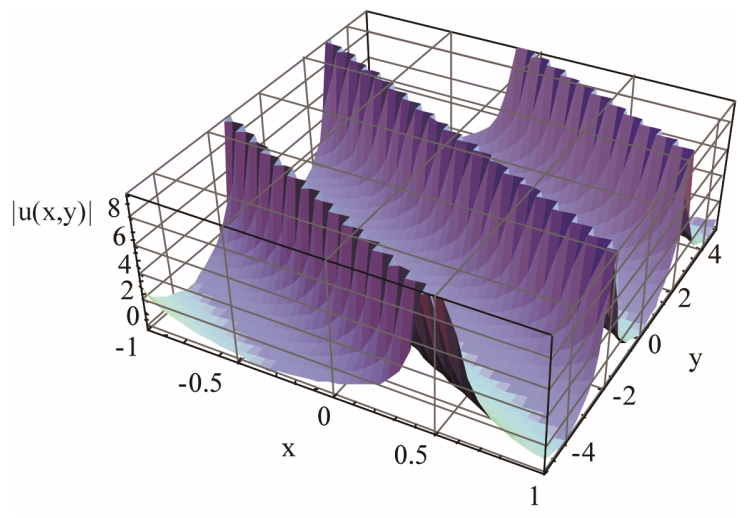

(b)

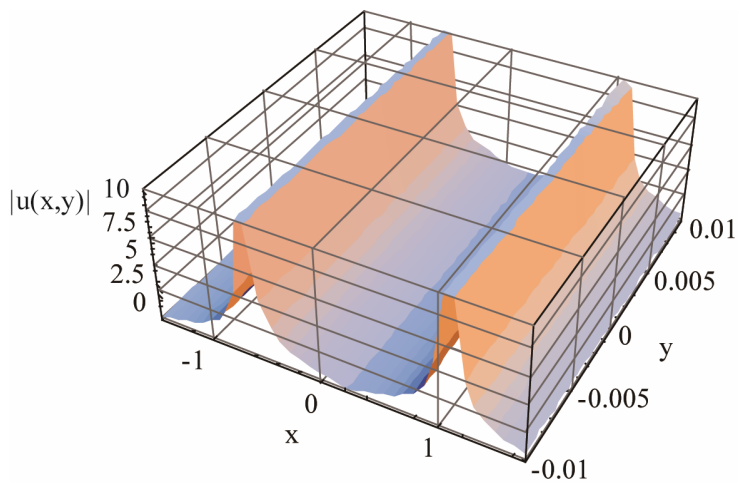

(d)

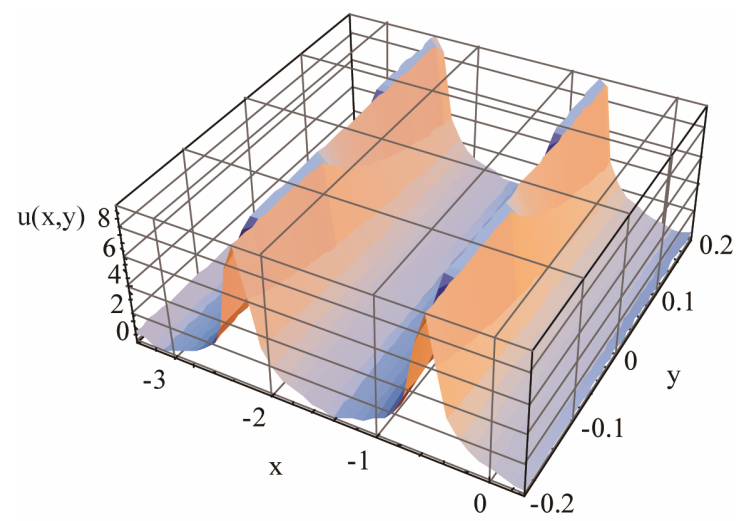

(e)

Figure 2. The solitary wave solution represented by Equations (34), (36), (38), (40) and (42) for various values $\alpha=1, \beta=2, l=$ $0.9, n=2, \Omega=3 ; p=0.5, t=7$, for Figures 2(a), 2(b), 2(c), 2(d), 2(e) respectively.

cially as they deal with the equations of non-linear coefficients fixed and transactions variable, which explain the phenomena, physical and in the various sciences. In my view this lack of interest due to the ease of the abovementioned methods.

\section{Acknowledgements}

It is a pleasure to thank the referee for critical comments on this work.

\section{REFERENCES}

[1] R. Hirota, "Exact Solution of the Korteweg-de VriesEquation for Multiple Collisions of Solitons," Physical Review Letters, Vol. 27, No. 18, 1971, pp. 1192-1194. doi:10.1103/PhysRevLett.27.1192

[2] C. Rogers and W. Shadwick, "Bäcklund Transformations and Their Applications," Academic Press, New York, 1982.

[3] M. J. Ablowitz and P. A. Clarkson, "Solitons, Nonlinear Evolution Equations and Inverse Scattering," Cambridge 
University Press, Cambridge, 1991. doi:10.1017/CBO9780511623998

[4] J. Weiss, "The Panlevé Property for Partial Differential Equations. II: Bäcklund Transformation, Lax Pairs, and the Schwarzian Derivative," Journal of Mathematical Physics, Vol. 24, No. 6, 1983, pp. 1405-1413. doi: $10.1063 / 1.525875$

[5] Z. Y. Yan, "An Improved Algebra Method and Its Applications in Nonlinear Wave Equations," Chaos, Solitons \& Fractals, Vol. 21, No. 4, 2004, pp. 1013-1021.

[6] G. T. Liu and T. Y. Fan, "New Applications of Developed Jacobi Elliptic Function Expansion Methods," Physics Letters A, Vol. 345, No. 1-3, 2005, pp. 161-166. doi:10.1016/j.physleta.2005.07.034

[7] O. H. El-Kalaawy, "Exact Solitary Solution of Schamel Equation in Plasmas with Negative Ions," Physics Plasmas, Vol. 18, No. 11, 2011, pp. 112302-112309. doi:10.1063/1.3657422

[8] M. L. Wang, "Exact Solutions for a Compound KdVBurgers Equation," Physics Letters A, Vol. 213, No. 5-6, 1996, pp. 279-287. doi:10.1016/0375-9601(96)00103-X

[9] H. J. S. Dorren, "On the Integrability of Nonlinear Partial Differential Equations," Journal of Mathematical Physics, Vol. 40, No. 4, 1999, pp. 1966-1976. doi: $10.1063 / 1.532843$

[10] O. H. EL-Kalaawy, "Exact Soliton Solutions for Some Nonlinear Partial Differential Equations," Chaos, Solitons \& Fractals, Vol. 14, No. 4, 2002, pp. 547-552. doi:10.1016/S0960-0779(01)00217-X

[11] O. H. El-Kalaawy and R. S. Ibrahim, "Exact Solutions for Nonlinear Propagation of Slow Ion Acoustic Monotonic Double Layers and a Solitary Hole in a Semirelativistic Plasma," Physics Plasmas, Vol. 15, No. 7, 2008, Article ID: 072303. doi:10.1063/1.2956336

[12] M. A. Abdou, "Further Improved F-Expansion and New Exact Solutions for Nonlinear Evolution Equations," Nonlinear Dynamics, Vol. 52, No. 3, 2008, pp. 277-288. doi:10.1007/s11071-007-9277-3

[13] Y. J. Ren, S. T. Liu and H. Q. Zhang, "On a Generalized Improved F-Expansion Method," Communications in Theoretical Physics, Vol. 45, No. 1, 2006, pp. 15-28. doi:10.1088/0253-6102/45/1/003

[14] E. G. Fan, "Uniformly Constructing a Series of Explicit Exact Solutions to Nonlinear Equations in Mathematical Physics," Chaos, Solitons \& Fractals, Vol. 16, No. 5, 2005, pp. 819-839. doi:10.1016/S0960-0779(02)00472-1

[15] E. Yomba, "The Extended Fans Sub-Equation Method and Its Application to KdV-MKdV, BKK and Variant Boussinesq Equations," Physics Letters A, Vol. 336, No. 6, 2005, pp. 463-476.

[16] D. H. Feng and G. X. Luo, "The Improved Fan Sub Equation Method and Its Application to the SK Equation," Applied Mathematics and Computation, Vol. 215, No. 5, 2009, pp. 1949-1967. doi:10.1016/j.amc.2009.07.045

[17] S. Zhang and H. Q. Zhang, "Fan Sub-Equation Method for Wick-Type Stochastic Partial Differential Equations," Physics Letters A, Vol. 374, No. 41, 2010, pp. 4180-4187. doi:10.1016/i.physleta.2010.08.023
[18] A. Borhanifar, M. M. Kabir and L. M. Vahdat, "New Periodic and Soliton Wave Solutions for the Generalized Zakharov System and (2+1)Dimensional Nizhink Novikov-Veselov System," Chaos, Solitons \& Fractals, Vol. 42, No. 3, 2009, pp. 1646-1654. doi:10.1016/j.chaos.2009.03.064

[19] W. Malfliet, "Solitary Wave Solutions of Nonlinear Wave Equations," American Journal of Physics, Vol. 60, No. 7, 1992, pp. 650-654. doi:10.1119/1.17120

[20] E. Fan, "Extended tanh-Function Method and Its Applications to Nonlinear Equations," Physics Letters A, Vol. 277, No. 4-5, 2000, pp. 212-218. doi:10.1016/S0375-9601(00)00725-8

[21] E. Fan and Y. C. Hon, "Applications of Extended tanh Method to Special Types of Nonlinear Equations," Applied Mathematics and Computation, Vol. 141, No. 2-3, 2003, pp. 351-358. doi:10.1016/S0096-3003(02)00260-6

[22] W. Malfiet and W. Hereman, "The tanh Method I: Exact Solutions of Nonlinear Evolution and Wave Equations," Physica Scripta, Vol. 54, No. 6, 1996, pp. 563-568. doi:10.1088/0031-8949/54/6/003

[23] A. M. Wazwaz, "The tanh and the Sinecosine Methods for the Complex Modified KdV and the Generalized KdV Equation," Computers \& Mathematics with Applications, Vol. 49, No. 7-8, 2005, pp. 1101-1112. doi:10.1016/j.camwa.2004.08.013

[24] Q. M. Al-Mdallal and M. I. Syam, "Sinecosine Method for Finding the Soliton Solutions of the Generalized Fifth-Order Nonlinear Equation," Chaos, Solitons \& Fractals, Vol. 33, No. 5, 2007, pp. 1610-1617. doi:10.1016/j.chaos.2006.03.039

[25] J. H. He and X. H. Wu, "Variational Iteration Method: New Development and Applications," Computers \& Mathematics with Applications, Vol. 54, No. 7-8, 2007, pp. 881-894. doi:10.1016/j.camwa.2006.12.083

[26] J. Biazar and H. Ghazvini, "Homotopy Perturbation Transform Method for Solving Hyperbolic Partial Differential Equations," Computers \& Mathematics with Applications, Vol. 56, No. 2, 2008, pp. 453-458. doi:10.1016/j.camwa.2007.10.032

[27] M. Wang, X. Li and J. Zhang, "The $\left(G^{\prime} / G\right)$ Expansion Method and Traveling Wave Solutions of nonlinear evolution Equations in Mathematical Physics," Physics Letters A, Vol. 372, No. 4, 2008, pp. 417-423. doi:10.1016/j.physleta.2007.07.051

[28] J. Zhang, X. Wei and Y. J. Lu, “A Generalized $\left(G^{\prime} / G\right)$ Expansion Method and Its Applications," Physics Letters A, Vol. 372, 2008, pp. 36-53.

[29] B. Zheng, "Travelling Wave Solutions of Two Nonlinear Evolution Equations by Using the $\left(G^{\prime} / G\right)$-Expansion Method," Applied Mathematics and Computation, 2010. doi:10.1016/j.mac.2010.12.052

[30] A. Borhanifar and M. M. Kabir, "New Periodicand Soliton Solutions by Application of Exp-Function Method for Linear Evolution Equations," Journal of Computational and Applied Mathematics, Vol. 229, No. 1, 2009, pp. 
158-167. doi:10.1016/j.cam.2008.10.052

[31] S. A. El-Wakil, M. A. Abdou and A. Hendi, "New Periodic Wave Solutions via Exp-Function Method," Physics Letters A, Vol. 372, No. 6, 2008, pp. 830-840. doi:10.1016/j.physleta.2007.08.033

[32] H. Zhao and C. Bai, "New Doubly Periodic and Multiple Soliton Solutions of the Generalized (3+1)Dimensional Kadomtsev-Petviashvilli Equation with Variable Coefficients," Chaos, Solitons \& Fractals, Vol. 30, No. 1, 2006, pp. 217-226. doi:10.1016/j.chaos.2005.08.148

[33] M. A. Abdou and A. Elhanbaly, "Construction of Periodic and Solitary Wave Solutions by the Extended Jacobi Elliptic Function Expansion Method," Communications in Nonlinear Science and Numerical Simulation, Vol. 12, No. 7, 2007, pp. 1229-1241. doi:10.1016/j.cnsns.2006.01.013

[34] Z. Yan, "Abudant Families of Jacobi Elliptic Function Solutions of the (2+1)-Dimensional Integrable DaveyStewartson Equation via a New Method," Chaos, Solitons \& Fractals, Vol. 18, No. 2, 2003, pp. 299-309. doi:10.1016/S0960-0779(02)00653-7

[35] W. X. Ma and B. Fuchssteiner, "Explicit and Exact Solutions to a Kolmogorov-Petrovskii-Piskunov Equation," International Journal of Non-Linear Mechanics, Vol. 31, No. 3, 1996, pp. 329-338. doi:10.1016/0020-7462(95)00064-X

[36] W. X. Ma, "Travelling Wave Solutions to a Seventh Order Generalized KdV Equation," Physics Letters A, Vol. 180, No. 3, 1993, pp. 221-224. doi:10.1016/0375-9601(93)90699-Z

[37] W. X. Ma, H. Y. Wu and J. S. He, "Partial Differential Equations Possessing Frobenius Integrable Decompositions," Physics Letters A, Vol. 364, No. 1, 2007, pp. 2932. doi:10.1016/j.physleta.2006.11.048

[38] W. X. Ma and J. H. Lee, "A Transformed Rational Func- tion Method and Exact Solutions to the $(3+1)$ Dimensional Jimbo-Miwa Equation," Chaos, Solitons \& Fractals, Vol. 42, No. 3, 2009, pp. 1356-1363. doi:10.1016/j.chaos.2009.03.043

[39] W. X. Ma, T. W. Huang and Y. Zhang, "A Multiple exp-Function Method for Nonlinear Differential Equations and Its Application," Physica Scripta, Vol. 82, No. 6, 2010, Article ID: 065003. doi:10.1088/0031-8949/82/06/065003

[40] W. X. Ma, Y. Zhang, Y. Tang and J. Tu, "Hirota Bilinear Equations with Subspaces of Solutions," Applied Mathematics and Computation, Vol. 218, No. 13, 2012, pp. 7174-7183. doi:10.1016/j.amc.2011.12.085

[41] F. C. You, T. C. Xia and J. Zhang, "Frobenius Integrable Decompositions for Two Classes of Nonlinear Evolution Equations with Variable Coefficients," Modern Physics Letters B, Vol. 23, No. 12, 2009, pp. 1519-1524. doi:10.1142/S0217984909019764

[42] Z. Huang, "On Cauchy Problems for the RLW Equation in Two Space Dimensions," Applied Mathematics and Mechanics, Vol. 23, 2002, pp. 159-164.

[43] Y. Shang and P. Niu, "Explicit Exact Solutions for the RLW Equation and the SRLW Equation in Two Space Dimensions," Applied Mathematics, Vol. 11, No. 3, 1988, pp. 1-5.

[44] T. Kawahara, K. Araki and S. Toh, "Interactions of TwoDimensionally Localized Pulses of the Regularized-LongWave Equation," Physica D: Nonlinear Phenomena, Vol. 59, No. 1-3, 1992, pp. 79-89. doi:10.1016/0167-2789(92)90207-4

[45] A. Davey and K. Stewartson, "On Three-Dimensional Packets of Surface Waves," Proceedings of the Royal Society A, Vol. 338, No. 1613, 1974, pp. 101-110. doi:10.1098/rspa.1974.0076 


\section{Appendix}

Relation between values of $\left(q_{0}, q_{2}, q_{4}\right)$ and corresponding $f(\zeta)$ in ODE $\left(f^{\prime}\right)^{2}=q_{0}+q_{2} f^{2}(\zeta)+q_{4} f^{4}(\zeta)$.

\begin{tabular}{|c|c|c|c|}
\hline$q_{0}$ & $q_{2}$ & $q_{4}$ & $F(\zeta)$ \\
\hline 1 & $1-m^{2}$ & $m^{2}$ & $s n \zeta, c d \zeta=\frac{c n \zeta}{d n \zeta}$ \\
\hline $1-m^{2}$ & $2 m^{2}-1$ & $-m^{2}$ & $c n \zeta$ \\
\hline$m^{2}-1$ & $2-m^{2}$ & -1 & $d n \zeta$ \\
\hline$m^{2}$ & $-1-m^{2}$ & 1 & $n s \zeta, d c \zeta=\frac{d n \zeta}{c n \zeta}$ \\
\hline$-m^{2}$ & $2 m^{2}-1$ & $1-m^{2}$ & $n c \zeta$ \\
\hline-1 & $2-m^{2}$ & $m^{2}-1$ & $n d \zeta$ \\
\hline 1 & $2-m^{2}$ & $1-m^{2}$ & $s c \zeta=\frac{s n \zeta}{c n \zeta}$ \\
\hline 1 & $2 m^{2}-1$ & $-m^{2}\left(-1-m^{2}\right)$ & $s d \zeta=\frac{s n \zeta}{d n \zeta}$ \\
\hline $1-m^{2}$ & $2-m^{2}$ & 1 & $\operatorname{cs} \zeta=\frac{c n \zeta}{s n \zeta}$ \\
\hline$-m^{2}\left(1-m^{2}\right)$ & $2 m^{2}-1$ & 1 & $d s \zeta=\frac{d n \zeta}{s n \zeta}$ \\
\hline 1 & $m^{2}-2$ & $m^{2}$ & $s n \zeta \quad c n \zeta$ \\
\hline$\overline{4}$ & $\overline{2}$ & $\overline{4}$ & $\overline{1 \pm d n \zeta}, \overline{\sqrt{1-m^{2}} \pm d n \zeta}$ \\
\hline$m^{2}$ & $m^{2}-2$ & $m^{2}$ & $s n \zeta+\mathrm{i} c n \zeta, m s n \zeta$ \\
\hline$\overline{4}$ & $\overline{2}$ & $\overline{4}$ & $s n_{\zeta}+1 c n \zeta, \overline{1 \pm d n \zeta}$ \\
\hline 1 & $1-2 m^{2}$ & 1 & $m \operatorname{sn} \zeta+\mathrm{i} d n \zeta, \frac{c n \zeta}{}$ \\
\hline$\overline{4}$ & 2 & $\overline{4}$ & ds \\
\hline$m^{2}-1$ & $m^{2}+1$ & $m^{2}-1$ & $d n \zeta$ \\
\hline$\overline{4}$ & $\overline{2}$ & $\overline{4}$ & $\overline{1 \pm m s n \zeta}$ \\
\hline $1-m^{2}$ & $m^{2}+1$ & $1-m^{2}$ & $c n \zeta$ \\
\hline$\overline{4}$ & $\overline{2}$ & $\overline{4}$ & $\overline{1 \pm \operatorname{sn} \zeta}$ \\
\hline$\left(1-m^{2}\right)^{2}$ & $m^{2}+1$ & -1 & $m c s \zeta \pm d n \zeta$ \\
\hline 4 & $\overline{2}$ & $\overline{4}$ & \\
\hline$\underline{1}$ & $m^{2}+1$ & $\left(1-m^{2}\right)^{2}$ & $s n \zeta$ \\
\hline$\overline{4}$ & $\overline{2}$ & 4 & $\overline{c n \zeta \pm d n \zeta}$ \\
\hline
\end{tabular}

